\title{
Usefulness of porA sequencing in distinguishing sporadic and linked cases of serogroup B invasive meningococcal disease in Suffolk, United Kingdom, December 2009 to January 2010
}

A Varadarajan (abrajan@doctors.org.uk) ${ }^{1}$, T Sundkvist ${ }^{1}$, N Jayatilleke $^{1}$, C Williams $^{1}$, S J Gray ${ }^{2}$, A D Carr², E B Kaczmarski ${ }^{2}$

1. Norfolk, Suffolk and Cambridgeshire Health Protection Unit, Health Protection Agency, Ipswich, Suffolk, United Kingdom

2. Meningococcal Reference Unit, Health Protection Agency, Manchester, United Kingdom

Citation style for this article:

Varadarajan A, Sundkvist T, Jayatilleke N, Williams C, Gray SJ, Carr AD, Kaczmarski EB. Usefulness of porA sequencing in distinguishing sporadic and linked cases of serogroup B invasive meningococcal disease in Suffolk, United Kingdom, December 2009 to January 2010. Euro Surveill. 2011;16(4):pii=19775. Available online: http://www.eurosurveillance.org/ViewArticle.aspx?Articleld=19775

Article published on 27 January 2011

A cluster of three fatal cases of invasive meningococcal disease due to Neisseria meningitidis serogroup B in a town in Suffolk, United Kingdom, during December 2009 to January 2010 was reported to the local Health Protection Unit. This paper describes the investigation undertaken to identify any potential epidemiological links among the cases, to determine if this was an outbreak and to consider whether to implement community-wide interventions and control measures. Case epidemiological information in addition to serogroup and genosubtyping (porA gene sequencing) data of the infecting organism was gathered on all cases in this reported cluster. Genosubtyping was also retrospectively requested for all serogroup $B$ cases confirmed in Suffolk during 2009. Extensive investigation failed to establish an epidemiological link among the cluster of fatal cases of serogroup B invasive meningococcal disease in Suffolk. By demonstrating a number of distinct strains, the genosubtyping of isolates proved to be useful in the public health management of this incident by serving to exclude a community outbreak and preventing unnecessary mass chemoprophylaxis.

\section{Introduction}

Invasive meningococcal disease is caused by Neisseria meningitidis, which is a normal inhabitant of the human nasopharynx. Transmission from person to person occurs by contact with droplets or secretions from the upper respiratory tract. Meningococci are classified into serogroups based on capsular polysaccharide antigens: there are at least 13 serogroups, of which serogroups $A, B, C, W_{135}$ and $Y$ account for the majority of the invasive meningococcal disease worldwide [1]. In the past, serogroups $B, C$ and $Y$ were the most common in the United Kingdom (UK). Following the introduction of the meningococcal serogroup $C$ conjugate vaccine into the UK routine immunisation programme in November 1999, the number of cases of invasive meningococcal disease caused by serogroup C fell by over $90 \%$ [2]. Currently, serogroup B strains account for around $90 \%$ of laboratory-confirmed cases submitted to the Health Protection Agency.

Invasive meningococcal disease, although relatively rare, is a life-threatening infection with case fatality rates of about $10 \%[3,4]$. The annual rate of invasive disease in the United Kingdom (UK) ranges from two to six per 100,000 population, with a higher incidence in the winter months. The incidence of meningococcal disease is highest in infants under one year of age, followed by children aged one to five years. The next highest incidence is seen in young people aged 15 to 19 years $[1,3]$.

Between 14 December 2009 and 5 January 2010 the local Health Protection Unit in the county of Suffolk, United Kingdom, received reports of four fatal cases of suspected meningococcal disease associated with a Suffolk town. According to mid-2007 estimates, the population of the town was 121,047 and that of Suffolk was 709,409 [5].

All cases were notified within 24 hours of onset of symptoms. The local Health Protection Unit launched an investigation for potential epidemiological links between the cases, to identify if it might be an outbreak and to consider whether to implement interventions and control measures.

The aim of this paper is to demonstrate the usefulness of porA sequencing in distinguishing sporadic and linked cases in the investigation of a cluster of fatal cases of serogroup B invasive meningococcal disease.

\section{Methods}

We designated this group of cases as a cluster, in line with the United States Centers for Disease Control and Prevention (CDC) definition of a cluster as 'an aggregation of cases of a disease or other health-related conditions which are closely grouped in time and place' 
[6]. This definition does not assume that the number of cases is more than expected, nor that expected number is known.

\section{Case definition}

Confirmed case

A confirmed case was defined as an individual with a clinical presentation of invasive meningococcal disease, of any age with a place of residence or overnight stay (during the seven-day incubation period) in the local authority area of the town in Suffolk, with onset of illness on or after 1 December 2009 and with a laboratory confirmation of serogroup $B$.

\section{Probable case}

A probable case was defined as an individual with a clinical presentation of invasive meningococcal disease (without laboratory confirmation of serogroup B), of any age, with a place of residence or overnight stay (during the seven-day incubation period) in the local authority area of the town in Suffolk and with onset of illness on or after 1 December 2009.

\section{Clinical presentation}

The early symptoms of meningococcal disease may be non-specific and different in infants and older children. Symptoms in infants include fever, floppiness, highpitched crying and sometimes vomiting. Older children and adults may present with fever, increasing headache, neck stiffness, photophobia, nausea and vomiting. Some may present with a distinctive petechial rash.

\section{Case identification and investigation}

The first fatal case was a young child (Case 1) with fulminant septicaemia, who was notified to the local Health Protection Unit on 14 December 2009 as a probable case of invasive meningococcal disease. On 31 December 2009 two further fatal probable cases (Cases 2 and 3 , both young children) were notified to the local Health Protection Unit. All three cases were from the same town in Suffolk. Investigation was undertaken to identify if there was any link between the cases. A further fatal case (Case 4) in an adult was reported to the Health Protection Unit on 5 January 2010. This case had died abroad but had travelled during the sevenday incubation period from the same town as the other fatal cases.

The cluster was investigated for any epidemiological links that might indicate local transmission pathways. Clinical information and the movements of the cases in the seven days before their onset of illness were gathered to determine any spatio-temporal links. We calculated the three-month (October-December 2009) incidence per 100,000 population in the town for children in the 0-4-years age group (as the three initial cases were all below four years of age) to help determine whether an outbreak (more cases than expected) was occurring. Overall incidence and subtype-specific incidence were calculated separately. Only the first case in any household was used in the calculation of local incidences. The local authority boundary of the town was used as the population boundary for the incidence calculations. The UK guidelines on meningococcal disease discuss the criteria for implementing community-level interventions in a defined population, when an age-specific incidence exceeds 40 per 100,000 population [1].

\section{Microbiological investigations}

No ante-mortem clinical samples were available for Case 1; however, a post-mortem blood sample was taken for polymerase chain reaction (PCR) investigation (carried out at Great Ormond Street Hospital, London). DNA extracted from the blood sample was subsequently forwarded to Meningococcal Reference Unit for genosubtyping (porA gene sequencing). Genogrouping was carried out by TaqMan real-time siaD PCR assay following initial TaqMan real-time ctrA PCR assay screening [7].

Oropharyngeal samples for culture and peripheral blood samples for culture and PCR were taken from Cases 2 and 3. Results of the microbiological investigation of the fourth case were requested from the country where the case died.

\section{Genosubtyping}

The Meningococcal Reference Unit provided genosubtype information for three of the cluster cases. Retrospective genosubtyping of all the cases of serogroup B invasive meningococcal disease confirmed in Suffolk during 2009 was also carried out, to compare with those of the cluster and identify any predominant genosubtype in the community. There were a total of 19 cases of serogroup B invasive meningococcal disease in Suffolk in 2009, including the two cases from the cluster (for which the genosubtype was available). Therefore samples from a further 17 cases were investigated retrospectively by the Unit, seven of which were confirmed by PCR only (culture negative). Although all case isolates are routinely characterised by porA gene sequencing, for patients whose infection is confirmed by PCR alone, the N. meningitidis genosubtype is determined only in specific instances of public health interest, as the assay is difficult.

\section{Results}

\section{Case investigation and} microbiological investigation

The first fatal case (Case 1) notified on 14 December 2009 was appropriately managed as a single case of meningococcal disease according to national guidelines. The $N$. meningitidis serogroup for this case was not known in the early stages of investigation as clinical samples were not available; however PCR investigation of post-mortem samples later confirmed this as serogroup B. Case 2 was confirmed as being infected with N. meningitidis serogroup B by PCR.

The clinical presentation of Case 3, who had an underlying condition, was not clinically typical of invasive meningococcal disease. Blood cultures taken 
ante-mortem grew Gram-negative cocci. The organism was initially thought to be $N$. meningitidis but was later identified as Veillonella atypica, which is a Gramnegative coccus and strict anaerobe. The hospital laboratory considered that this was probably a contaminant. Case 3's PCR result (from a blood sample) and other cultures were negative for $N$. meningitidis. It was concluded that Case 3 did not have invasive meningococcal disease and therefore was excluded from the cluster.

A laboratory in the country where the fourth case died kindly provided confirmation of the case's serogroup (serogroup B), which was determined by PCR (from a skin biopsy).

The close contacts of all four cases were identified and given prophylaxis according to the UK national guidelines.

\section{Genosubtyping data}

Table 1 shows that Cases 1 and 2 were indistinguishable by porA gene sequencing, but no epidemiological links could be established between them. For Case 4

\section{TABLE 1}

Serogroup and genosubtypes of the cluster of fatal cases of invasive meningococcal disease in a town in Suffolk, United Kingdom, December 2009-January $2010(n=3)^{a}$

\begin{tabular}{|l|c|c|c|}
\hline $\begin{array}{l}\text { Case } \\
\text { number }\end{array}$ & $\begin{array}{c}\text { Age or } \\
\text { age range } \\
\text { (years) }\end{array}$ & Serogroup & porA genosubtype \\
\hline 1 & $0-4$ & $\mathrm{~B}$ & $\mathrm{VR} 1=19-1, \mathrm{VR} 2=15-11, \mathrm{VR} 3=36$ \\
\hline 2 & $0-4$ & $\mathrm{~B}$ & $\mathrm{VR} 1=19-1, \mathrm{VR} 2=15-11, \mathrm{VR} 3=36$ \\
\hline $4^{\mathrm{b}}$ & Mid-40s & $\mathrm{B}$ & $\mathrm{VR} 1=7$ \\
\hline
\end{tabular}

VR: variable region.

a Case 3 was excluded from the cluster as this case did not have invasive meningococcal disease.

${ }^{b}$ Only data on the porA VR1 were available for Case 4, due to limited availability of clinical material (skin biopsy).

\section{TABLE 2}

Data on porA genosubtypes of all typeable cases of serogroup B invasive meningococcal disease in Suffolk, United Kingdom, $2009(\mathrm{n}=17)^{\mathrm{a}}$

\begin{tabular}{|l|c|}
\hline $\begin{array}{l}\text { Number of cases } \\
\text { per genosubtype }\end{array}$ & porA genosubtype \\
\hline 5 & $\mathrm{VR}_{1}=22, \mathrm{VR} 2=14, \mathrm{VR} 3=36$ \\
\hline 4 & $\mathrm{VR} 1=22, \mathrm{VR} 2=9, \mathrm{VR} 3=35-1$ \\
\hline 3 & $\mathrm{VR} 1=19-1, \mathrm{VR} 2=15-11, \mathrm{VR} 3=36$ \\
\hline 1 & $\mathrm{VR} 1=22, \mathrm{VR} 2=14-26, \mathrm{VR} 3=36$ \\
\hline 1 & $\mathrm{VR} 1=21, \mathrm{VR} 2=16, \mathrm{VR}_{3}=37-1$ \\
\hline 1 & $\mathrm{VR} 1=7-2, \mathrm{VR} 2=4, \mathrm{VR} 3=37$ \\
\hline 1 & $\mathrm{VR} 1=5-1, \mathrm{VR} 2=10-1, \mathrm{VR} 3=36-2$ \\
\hline 1 & $\mathrm{VR} 1=7-1, \mathrm{VR} 2=1, \mathrm{VR} 3=35-1$ \\
\hline
\end{tabular}

VR: variable region.

a Of the 19 serogroup B invasive meningococcal cases in Suffolk in 2009 , porA sequence subtype data were available for 17 cases. The genosubtype determined for Cases 1 and 2 of the cluster of fatal cases in December 2009 to January 2010 is highlighted. (the adult fatal case), data were only available for the porA VR1, due to limited availability of clinical material (skin biopsy). However, this was sufficient to show that Case 4 was distinct and was not linked to Cases 1 and 2.

A total of 17 samples from serogroup B invasive meningococcal cases in Suffolk in 2009 were retrospectively analysed to determine their genosubtype. Data were available for 15 samples only, as two samples were not typeable. The genosubtype information on 17 serogroup B invasive meningococcal cases (two from the 2009 cluster and 15 from retrospective analysis) for 2009 in Suffolk is shown in Table 2, which lists the porA variable region (VR) data. This clearly established that the porA subtype VR1=19-1, VR2 $=15-11, V_{3}=36$ (present in Cases 1 and 2 of the cluster of fatal cases) was one of the more common genosubtypes circulating in the community. This specific porA subtype represented $18 \%$ (three of 17 ) of all typeable serogroup $B$ invasive meningococcal isolates in Suffolk and was the fourth most common genosubtype in England and Wales in 2009 (unpublished 2010 data from the Meningococcal Reference Unit).

Epidemiological analysis

The three-month (October to December 2009) incidence of serogroup $B$ invasive meningococcal disease in children aged $0-4$ years in Suffolk was 37 per 100,000 population. The incidence of the disease caused by the $N$. meningitidis genosubtype $\mathrm{VR} 1=19-1, \mathrm{VR} 2=15-11$, $V_{R}=36$ in the same age group for the same period was 24.6 per 100,000 population. This porA genosubtype, identified for Cases 1 and 2 of the cluster of fatal cases, was one of the common porA subtypes among isolates from cases of serogroup $B$ invasive meningococcal disease from England and Wales in 2009. Retrospective porA sequencing of DNA from all the available Suffolk 2009 case material confirmed one other patient with a N. meningitidis porA subtype indistinguishable to that of Cases 1 and 2 of the cluster; however, this was a non-fatal case reported in the summer of 2009, in another part of the county.

There were two other cases of serogroup B invasive meningococcal disease in late December 2009 in Suffolk (in different towns to the cluster of fatal cases): both had a common porA subtype but were not epidemiologically linked and the porA subtype was distinct from that of Cases 1 and 2 of the cluster.

\section{Control measures}

The main control measure was to identify close contacts, as recommended in the UK national guidelines, and arrange for appropriate chemoprophylaxis. The guidelines recommend that the antibacterial agents rifampicin, ciprofloxacin and ceftriaxone be used to prevent secondary cases. Locally, rifampicin was used for persons aged under two years and ciprofloxacin for persons aged over two years. For pregnant or breastfeeding women, rifampicin or ceftriaxone are used. 
Rifampicin is the only antibiotic that is licensed in the UK for the purpose of chemoprophylaxis.

Local general practitioners and out-of-hours services including accident and emergency departments were advised to be on the alert for new cases of meningococcal disease. In addition, information on signs and symptoms of the disease, including advice to seek medical advice rapidly, was provided to the local community using the local media.

\section{Discussion and conclusions}

The identification of a community outbreak is a challenge, especially when considering community interventions where the population boundary will of necessity be arbitrary. The options for community interventions are limited in outbreaks of serogroup $B$ invasive meningococcal disease [8]. Community interventions include antibiotic prophylaxis and for serogroups $\mathrm{A}, \mathrm{C}, \mathrm{Y}$ or $\mathrm{W}_{135}$, vaccination against the relevant outbreak organism. There are no licensed serogroup $B$ vaccines currently available in the UK. Strain-specific outer membrane vesicle vaccines have been used in some countries such as New Zealand [9] and France [10] to control a national and regional epidemic of serogroup B meningococcal disease dominated by a single strain. Mass chemoprophylaxis has disadvantages: it can lead to potentially serious complications and development of resistance [11]. It could also result in the elimination of non-pathogenic Neisseria species, leading to reduced immunity against pathogenic species, and would not prevent the reintroduction of the outbreak strain after the prophylaxis $[12,13]$.

The UK guidelines recommend intervention in community outbreaks if the age-specific incidence in a three-month period is high and a pragmatic threshold for triggering intervention has been set. Targeted community interventions have been implemented in the UK in the past, where the age-specific incidence exceeded 40 per 100,000 population in serogroup C outbreaks of four or more cases [1].

The Communicable Diseases Network Australia guidelines define a community outbreak as three or more confirmed cases of a specific serosubtype in a threemonth period with an incidence rate of 10 per 100,000 population and explicitly states that mass chemoprophylaxis should not be used in community outbreaks [14]. Australia, like the UK, currently has no licensed serogroup B vaccines.

Similarly the US CDC guidelines on prevention and control of meningococcal disease state that currently there is no licensed serogroup B vaccine available in the US and mass chemoprophylaxis is not generally recommended for large outbreaks in the community due to the disadvantages of chemoprophylaxis [12]. The CDC recommends that in outbreaks involving limited populations (e.g. an outbreak in a single school), adminis- tration of chemoprophylaxis could be considered in serogroup B outbreaks [15].

The guidelines on community interventions for outbreaks of serogroup B are similar across countries and the options are fairly limited, as discussed above. Nevertheless, it is important to identify a community outbreak or rule out an outbreak in order to implement appropriate public health interventions. Meningococcal disease clusters can cause a great deal of public anxiety and media interest, especially when they involve fatal cases. In our cluster investigation, we found the incidence of disease caused by the $N$. meningitidis genosubtype VR1=19-1, VR2=15-11, VR3=36 (24.6 per 100,000 population) for children aged $0-4$ years was well below the trigger for any community interventions according to UK guidelines. It is important to note that the local authority boundary was chosen as an arbitrary geographical boundary (population boundary) for the incidence calculations.

Although epidemiological links are useful in identifying transmission pathways in clusters and outbreaks, molecular techniques to characterise the isolates provide extremely useful supporting information [16, 17]. In our investigation we found that genosubtyping (using porA gene sequencing) of the N. Meningitides, infecting cases in the town and the data on the incidence of the various genosubtypes of serogroup $B$ invasive meningococcal disease in England and Wales were useful in delivering an appropriate public health response. Although the two fatal cases in December 2009 were of indistinguishable genosubtype, this subtype was found to be one of the common strains and it was concluded that they were likely to be sporadic cases. Testing for additional antigens or other genes for variation (discrimination) was not routinely carried out at the Meningococcal Reference Unit. At the Unit, given the practicability and discrimination afforded by porA sequencing, developed for culture and nonculture material, this approach has been used for the investigation of clusters. Multilocus sequence typing (MLST) is currently not used routinely for the investigation of clusters, but has recently been agreed by the Health Protection Agency as the preferred second-line discrimination assay. Testing for other gene targets (such as ferric enterochelin receptor, fetA, and factor $\mathrm{H}$ binding protein, $\mathrm{fHBP}$ ) are under investigation but the practicality and economic feasibility of such tests remain to be determined.

It was determined that two non-fatal invasive meningococcal cases in Suffolk between 25 and 31 December 2009 had an indistinguishable porA gene sequence $(V R 1=22, V R 2=9, V R 3=35-1)$, but it was different to that of Cases 1 and 2. These non-fatal cases were from different parts of the county, with no epidemiological links. They were sporadic cases caused by one of the other common strains in the UK. The porA gene sequencing data on all typeable 2009 Suffolk invasive meningococcal cases obtained from the Meningococcal 
Reference Unit proved very useful in understanding the dynamic nature of different strains circulating in Suffolk. In the investigation of clusters, porA gene sequencing can be performed to differentiate between strains within the timescale required for public health action [14]. Sequencing of the porA gene was a very useful tool in the risk assessment of our investigation.

In conclusion, extensive investigation failed to establish an epidemiological link among the fatal cases of serogroup B invasive meningococcal disease in this reported cluster. The genosubtyping of the isolates proved important in the public health management of this incident by showing that the cases were not part of an outbreak with transmission links, in which mass chemoprophylaxis might have been considered.

\section{Acknowledgements}

We would like to thank all those who were part of the outbreak management team and all those who assisted in the investigation of this outbreak and implementation of control measures. We wish to thank the local National Health Service Trust hospital especially Dr Richard Kent in the microbiology team and the paediatric team who provided us with information during this investigation. We also wish to thank staff at the Meningococcal Reference Unit who kindly provided us with a very rapid porA gene sequencing service.

\section{References}

1. Health Protection Agency (HPA). Guidance for public health management of meningococcal disease in the UK. August 2006. Available from: http://www.hpa.org.uk/web/ HPAwebFile/HPAweb_C/1194947389261

2. Trotter CL, Andrews NJ, Kaczmarski EB, Miller E, Ramsay ME. Effectiveness of meningococcal serogroup $C$ conjugate vaccine 4 years after introduction. Lancet. 2004;364(9431):365-7.

3. Ramsay M, Kaczmarski E, Rush M, Mallard R, Farrington P, White J. Changing patterns of case ascertainment and trends in meningococcal disease in England and Wales. Commun Dis Rep Rev CDR Rev. 1997;7(4):R49-R54.

4. Caro IJ, Möller J, Getsios D, Coudeville L, El-Hadi W, Chevat $C$, et al. Invasive meningococcal disease epidemiology and control measures: a framework for evaluation. BMC Public Health. 2007;7:130.

5. Office for National Statistics (ONS) [Internet]. Population estimates for UK, England and Wales, Scotland and Northern Ireland - current datasets. ONS: London. [Accessed 22 Sep 2010]. Available from http://www.statistics.gov.uk/statbase/ product.asp?vlnk=15106

6. Centers for Disease Control and Prevention (CDC) [Internet]. CDC: Atlanta, Georgia. [Accessed 22 Sep 2010]. Available from: http://www.cdc.gov/reproductivehealth/EpiGlossary/glossary. htm

7. Guiver M, Borrow R. PCR diagnosis. In: Pollard AJ, Maiden $M C J$, editors. Meningococcal disease: methods and protocols. Humana Press: Totowa, New Jersey; 2001. p. 21-39.

8. Jardine A, Truman G, Sheppeard V, Gibbons D, Thomas J, Weston K. A community outbreak of meningococcal serogroup $B$ disease in western Sydney: the challenges of identification and significance. Commun Dis Intell. 2009;33(2):221-4.

9. Lennon D, Jackson C, Wong S, Horsfall M, Stewart J, Reid $\mathrm{S}$. Fast tracking the vaccine licensure process to control an epidemic of serogroup B meningococcal disease in New Zealand. Clin Infect Dis. 2009;49(4):597-605.

10. Rouaud P, Perrocheau A, Taha MK, Sesboue C, Forgues A, Parent Du Chatelet I, Levy-Bruhl D. Prolonged outbreak of B meningococcal disease in the Seine-Maritime department, France, January 2003 to June 2005. Euro Surveill. 2006;11(7). pii: 635. Available online: http://www.eurosurveillance.org/ ViewArticle.aspx?Articleld $=635$
11. Jackson LA, Alexander ER, DeBolt CA, Swenson PD, Boase J, McDowell MG, et al. Evaluation of the use of mass chemoprophylaxis during a school outbreak of enzyme type 5 serogroup B meningococcal disease. Pediatr Infect Dis J. 1996;15(11):992-8.

12. Kristiansen BE, Knapskog AB. Secondary prevention of meningococcal disease. BMJ. 1996;312(7031):591-2.

13. Yagupsky P, Ashkenazi S, Block C. Rifampicin-resistant meningococci causing invasive disease and failure of chemoprophylaxis. Lancet. 1993; 341(8853):1152-3.

14. Communicable Diseases Network Australia. Guidelines for the early clinical and public health management of meningococcal disease in Australia. October 2007. Available from: http:/l www.health.gov.au/internet/main/publishing.nsf/Content/BC 329B583B663546CA25736Do07674AA/\$File/meningococcalguidelines.pdf

15. Bilukha OO, Rosenstein N; National Center for Infectious Diseases, Centers for Disease Control and Prevention (CDC). Prevention and control of meningococcal disease. Recommendations of the Advisory Committee on Immunization Practices (ACIP). National Center for Infectious Diseases, Centers for Disease Control and Prevention (CDC). MMWR Recomm Rep. 2005;54(RR-7):1-21.

16. Centers for Disease Control and Prevention (CDC). Outbreaks of group B meningococcal disease--Florida, 1995 and 1997. MMWR Morb Mortal Wkly Rep. 1998;47(39):833-7.

17. Clarke SC, Diggle MA, Edwards GF. Multilocus sequence typing and porA gene sequencing differentiates strains of Neisseria meningitidis during case clusters. Br J Biomed Sci. 2002;59(3):160-2. 